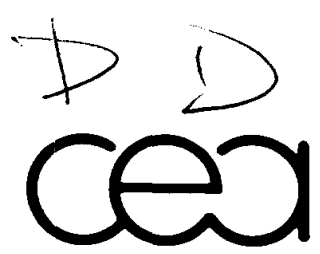

C.E. SACLAY DSM
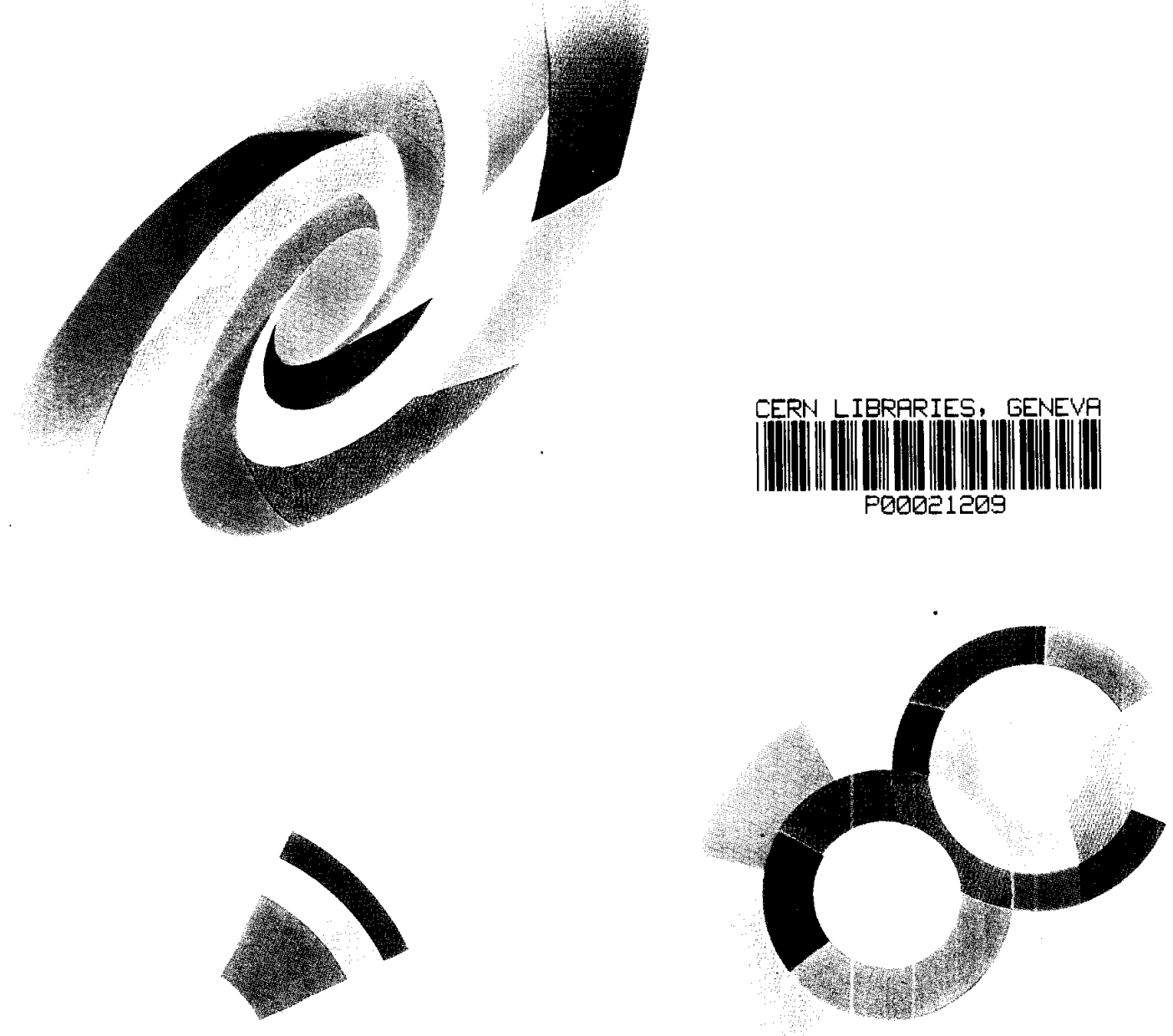

DAPNIA/SPP 94-02

LNGS - 94/88

January 1994

DARK MATTER SEARCH WITH CALCIUM

FLUORIDE CRYSTALS

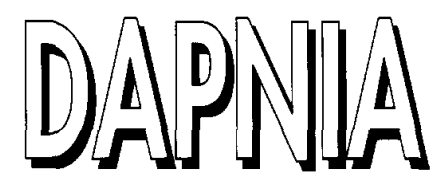

C. BACCI et al.

BPRS Collaboration 
Le DAPNIA (Département d'Astrophysique, de physique des Particules, de physique Nucléaire et de l'Instrumentation Associée) regroupe les activités du Service d'Astrophysique (SAp), du Département de Physique des Particules Elémentaires (DPhPE) et du Département de Physique Nucléaire (DPhN).

Adresse : $\quad$ DAPNIA, Bâtiment 141

CEA Saclay

F - 91191 Gif-sur-Yvette Cedex 


\title{
DARK MATTER SEARCH WITH CALCIUM FLUORIDE CRYSTALS
}

\author{
BPRS collaboration:
}

C. Bacci $^{a}$, P. Belli ${ }^{b}$, R. Bernabei ${ }^{b}$, C.J. Dai ${ }^{c *}$, W. Di Nicolantonio ${ }^{d}$, L.K. Ding ${ }^{c}$, E. Gaillard-Lecanu ${ }^{e}$, G. Gerbier ${ }^{e}$, Y. Giraud-Héraud ${ }^{f}$, H.H. Kuang ${ }^{c}$, A. Incicchitti ${ }^{d}$, J. Mallet ${ }^{e}$, L. Mosca ${ }^{e}$, D. Prosperi ${ }^{d}$, C. Tao ${ }^{f}$

a Dipartimento di Fisica, Università di Roma III and INFN, sez. Roma, I-00165 Rome, Italy

- Dipartimento di Fisica, Università di Roma "Tor Vergata" and INFN, sez. Roma II, I-00173 Rome, Italy

' IHEP, Chinese Academy, P.O. Box 918/3, Beijing 100099, China

d Dipartimento di Fisica, Università di Roma "La Sapienza" and INFN, sez. Roma, I-00165

Rome, Italy

- DSM/DAPNIA/SPP, C.E. Saclay, F-91191 Gif-sur-Yvette, France

$f$ Laboratoire de Physique Corpusculaire, Collège de France/ IN2P9 (CNRS), 11, place Marcellin Berthelot, F-75231 Paris, France

- presently at INFN Sezione Roma II, Italy

and

B. Chambon, V. Chazal, M. De Jésus, D. Drain, Y. Messous, C. Pastor

IPN Lyon, IN2P9 (CNRS), 49 Bd du 11 Novembre 1918, F-69622 Villeurbanne CEDEX, France

\begin{abstract}
A first result on dark matter direct search with calcium fluoride scintillators is presented. The low and high energy spectra are discussed together with the measurements of the fluorine and calcium recoil scintillation efficiencies. Exclusion plots for axial vector coupled WIMPs are derived and compared with previous measurements with $\mathrm{NaI}$ and Ge detectors.
\end{abstract}

\section{Introduction}

Many experimental efforts are in progress to search for cold dark matter candidates and various materials have been proposed and used both for coherent and spin-dependent interactions [ 1 to 7$]$. 
Our attention has been attracted by the possibility to use fluoride scintillating compounds as target-detectors as suggested in particular by J. Ellis and Flores in ref [8] where the large cross section on ${ }^{19} \mathrm{~F}$ (spin $1 / 2$ ) for axially coupled dark matter particles has been emphasized.

A large number of measurements has been performed by our group both in Gran Sasso (Laboratorio Nazionale del Gran Sasso, LNGS, Italy) and Modane Laboratories (Laboratoire Souterrain de Modane, LSM, France) with various fluoride scintillating detectors of different size and composition made by European and Chinese companies. We concentrated our efforts on $\mathrm{CaF}_{2}(\mathrm{Eu})$ because of the relatively large value of its light output ( $60 \%$ of $\mathrm{NaI}(\mathrm{Tl})$ ).

This paper presents an extensive study of $\mathrm{CaF}_{2}(\mathrm{Eu})$ crystals, including underground measurements of counting rates at high and low energy, determination of residual $\mathrm{U}$ and $\mathrm{Th}$ contaminations, measurements of scintillation efficiencies of $\mathrm{Ca}$ and $\mathrm{F}$ recoils with a neutron beam, and calculations of exclusion plots in the WIMP cross section/mass plane for axial vector coupled particles.

\section{Underground measurements}

\subsection{Low energy spectrum}

The data presented here have been collected at LNGS with a $0.37 \mathrm{~kg} \mathrm{CaF}_{2}(\mathrm{Eu})$ crystal by Quartz \& Silice, giving the most reduced counting rate and a very low energy threshold.

The crystal was placed inside a shield made by $1.5 \mathrm{~mm}$ of $\mathrm{Cd}, 15 \mathrm{~cm}$ of very low activity lead (less than $4 \mathrm{~Bq} / \mathrm{Kg}$ from ${ }^{210} \mathrm{~Pb}$ ) and $10 \mathrm{~cm}$ of selected very low activity copper; radon was removed by flushing gaseous nitrogen at about $1.2 \mathrm{~atm}$ through a special plastic material enveloping the shield. The crystal (3" diameter by 1 " length) was directly coupled to a very low activity 3" diameter EMI photomultiplier that has a measured rate of $\simeq 3 \mathrm{cpm}$ over $30 \mathrm{keV}$ in the LSM test facility.

A low activity $\mathrm{NaI}(\mathrm{Tl})$ detector $4 " \mathrm{x} 4$ " $\mathrm{x} 4$ " active volume is used as anti-Compton detector on the 3 " diameter surface not coupled with the photomultiplier of the $\mathrm{CaF}_{2}(\mathrm{Eu})$ detector. The scintillation light coming from the $\mathrm{NaI}(\mathrm{Tl})$ detector is seen through a bent light guide allowing to shield the $\mathrm{CaF}_{2}(\mathrm{Eu})$ crystal from the photomultiplier of the anti-Compton detector. From the measured single counting rate of the two detectors and the overlapping time in the anticoincidence module we derive that the random vetoes are 1 each 19 days of measurements.

For each event we record the time arrival, the ADC value and the pulse shape by a Lecroy transient digitizer, which allows a pulse shape analysis to reject photomultiplier noise. The comparison between the ADC value and the area of the pulse 
recorded by the transient digitizer allows to check the linearity of the ADC.

The detector has been calibrated at low energy using ${ }^{55} \mathrm{Fe}(5.9 \mathrm{keV})$ and ${ }^{109} \mathrm{Cd}$ $(22 \mathrm{keV}) \mathrm{X}$-rays; the iron calibration is shown in Fig. 1. The energy resolution is sigma $/ E=0.032+0.66 / \operatorname{sqrt}(E)$, with $\mathrm{E}$ in $\mathrm{keV}$.

Our experimental rate is shown in Fig. 2 and is referred to $(0.37 \times 32.2) \mathrm{kg}$.day. The quoted errors are statistical only. The presence of $U$ and $T h$ elements and their daughters in the crystal is responsible for a part of the residual experimental rate at low energy. However, as it will be shown in the following, the ${ }^{19} \mathrm{~F}$ is such a favourable nucleus for axially coupled particles that these measurements allow us to improve the exclusion plot already obtained with other targets.

\subsection{High energy spectrum: $U$ and Th contamination mea- surements}

Another 3 " by 1 " $\mathrm{CaF}_{2}(\mathrm{Eu})$ crystal from the Merck company was also studied in Modane. The crystal was coupled through a $10 \mathrm{~cm}$ straight light guide to the photomultiplier and protected from local activity by an appropriate shielding of lead and copper. For the low energy region, the counting rate was higher than for the Gran Sasso Q\&S crystal. In both crystals, the high energy behaviour is similar. The energy spectra exhibit structures around $1 \mathrm{MeV}$ (see Fig. 3) unexpected from gamma ray interactions in the crystal.

To determine the possible different origins of these pulses, each pulse was digitised and its decay time calculated. Fig. 4 shows the scatter plot of the decay time as a function of energy for the events of Fig. 3. Peaks in the energy distribution are clearly associated with higher decay times. Calibrations with gamma rays and $\alpha$ sources confirm that these events are caused by $\alpha$ particles generated inside the crystal. This discrimination allows to separate them from the background: the observed $\alpha$ rate is $(15 \pm 1) \mathrm{cpm} / \mathrm{kg}$ and $(0.28 \pm 0.02) \mathrm{cpm} / \mathrm{kg}$ for the Merck and Q\&S crystals, respectively.

Taking into account the scintillation efficiency for $\alpha$ 's relative to electrons, of about $15 \%$ [11], the mean energy of these $\alpha$ 's is found to be around $5 \mathrm{MeV}$. Their origin is attributed to decays of ${ }^{238} \mathrm{U}$ and ${ }^{232} \mathrm{Th}$ and their daughters. The observed spectrum has been fitted with the hypothesis of a mixture of $U$ and Th chains, with possible states out of equilibrium. Results of the fit for the Merck crystal (shown on Fig. 3) indicate that both chains are strongly out of equilibrium and that the $\alpha$ 's are coming in comparable proportions from $U(\simeq 40 \%)$ and $\mathrm{Th}(\simeq 60 \%)$ chains. The dominant elements in both chains are ${ }^{230} \mathrm{Th},{ }^{238} \mathrm{U}$, together with the ${ }^{226} \mathrm{Ra}$ to ${ }^{218} \mathrm{Po}$ section, and ${ }^{228} \mathrm{Th}$ to ${ }^{212} \mathrm{Po}$ section. 
Would the total number of $\alpha$ 's, in each chain, be distributed in configurations at equilibrium we obtain as "equivalent concentrations" $1.4 \mathrm{ppb}$ for ${ }^{238} \mathrm{U}$ and $6.6 \mathrm{ppb}$ for ${ }^{232} \mathrm{Th}$. An analogous fit for the Q\&S crystal gives as equivalent concentrations $0.05 \mathrm{ppb}$ for ${ }^{238} \mathrm{U}$ and $0.16 \mathrm{ppb}$ for ${ }^{232} \mathrm{Th}$.

Concentrations of $\mathrm{U} / \mathrm{Th}$ around $0.002 \mathrm{ppb}$ have already been obtained in low activity $\mathrm{NaI}(\mathrm{Tl})$ [12]. This indicates that improvements are possible at low energy in the case of $\mathrm{CaF}_{2}(\mathrm{Eu})$ where no particular selection relative to $\mathrm{U} / \mathrm{Th}$ contaminations has been done on the starting materials.

\section{Calibration of $\mathrm{CaF}_{2}(\mathrm{Eu})$ to $\mathrm{Ca}$ and $\mathrm{F}$ recoils}

\subsection{Method}

The same method developed to measure the ionization efficiency in semiconductors as $\mathrm{Si}[13]$ and $\mathrm{Ge}[14]$ has been used to study the light yield produced by ${ }^{19} \mathrm{~F}$ and $\mathrm{Ca}$ ions in $\mathrm{CaF}_{2}(\mathrm{Eu})$ scintillators. The crystal is bombarded with monoenergetic neutrons inducing elastic collisions. The kinetic energy of the recoil nucleus is deduced by detecting the scattered neutron at given angles. The light production is expressed in units of equivalent electron energy deposition producing the same scintillation. The $e^{-}$light response is linear in the energy region 5 to 15 electron equivalent $\mathrm{keV}$.

\subsection{Experimental set-up}

The experimental set-up is described in details in reference 15. The measurements are performed at the Tandem Facility of the C.E.N. of Bruyères-Le-Châtel. Neutrons are produced in the reaction $p+{ }^{7} \mathrm{Li} \rightarrow n+{ }^{7} \mathrm{Be}$ with proton energies of 3.0 and $3.6 \mathrm{MeV}$. The energy spread of the neutron beam is around $11 \mathrm{keV}(1 \sigma)$. A $10 \mathrm{~mm} \times 10 \mathrm{~mm} \times 4 \mathrm{~mm} \mathrm{CaF}_{2}(\mathrm{Eu})$ crystal of mass equal to $1.35 \mathrm{~g}$, is located 30 $\mathrm{cm}$ away from the neutron source. The elastically scattered neutrons are detected at five different angles by means of NE213 detectors set $80 \mathrm{~cm}$ away from the scintillator. The angular opening is close to $\Delta \theta= \pm 3.5^{\circ}$. For the neutron energies of interest here, the efficiency of these detectors is constant and equal to $55 \%$. Most of the uncorrelated events are removed by measuring the time of flight on neutron source- $\mathrm{CaF}_{2}(\mathrm{Eu})$ and $\mathrm{CaF}_{2}(\mathrm{Eu})$-neutron detectors flight paths together with pulse shape discrimination of the neutron hodoscope.

\subsection{Results}

The two electron equivalent energy spectra shown in Fig. 5 are obtained for recoil ions of ${ }^{19} F$ at $\mathrm{E}_{n}=1.3 \mathrm{MeV}$ and $\mathrm{Ca}$ and ${ }^{19} F$ at $\mathrm{E}_{n}=1.9 \mathrm{MeV}$. Scattered neutrons are detected at $\theta_{n}=115^{\circ}$. Events due to inelastic scattering could possibly distort the spectra. 
As far as collisions on $\mathrm{Ca}$ isotopes are concerned however, these events can be rejected by appropriate time of flight cuts. Considering the high energy of the first excited states, neutrons scattered in this process and elastically scattered neutrons have very different energies.

This is not true for inelastic scattering on ${ }^{19} \mathrm{~F}$ for which two excited levels are found at low energy $\left(E_{\text {exc }}=109.9 \mathrm{keV}\right.$ and $\left.197.2 \mathrm{keV}\right)$. Very few results on inelastic scattering of neutrons on ${ }^{19} \mathrm{~F}$ are found in the literature. The ratio $\frac{\sigma_{\text {inelast }}}{\sigma_{\text {elast }}}$ is estimated to be equal to $1 / 3$ by extrapolating the results already known [16]. This means that a fraction of about $1 / 3$ of ${ }^{19} \mathrm{~F}$ ions are inelastically scattered with recoiling energies $5 \%$ lower than elastically scattered ones. A simulation with GEANT shows that $12 \%$ of photons originating in decay from excited states, interact within the crystal, in particular through Compton scattering. This effect induces a tail in the high energy part of the peak. So the resulting shift due to the recoil of the inelastic part represents only $1 \%$ of the total energy.

The measured recoil energies in the crystal are reported in Fig. 6, in keV of equivalent electron energy, as a function of kinetic energies of ${ }^{19} \mathrm{~F}$ and $\mathrm{Ca}$ ions. The energy of $\mathrm{Ca}$ ions is computed from kinetic energies of $\mathrm{Ca}$ isotopes and weighted by the isotopic abundance ratios. Two curves are clearly seen for ${ }^{19} F$ and Ca nuclei. It confirms the properties of scintillation process for alkali-halide scintillators [17] at a given energy : the bigger the mass of the incident ion, the bigger is the amount of energy lost in interactions that do not generate light. For a given $Z$, the fraction of energy into scintillation $\left(\alpha(E)=\frac{d E_{\text {meas }}}{d E_{\text {recoil }}}\right)$ does not depend on the energy, within our errors, and is equal to $6.9 \pm 0.5 \%$ and $4.9 \pm 0.5 \%$ for ${ }^{19} \mathrm{~F}$ and $\mathrm{Ca}$ nuclei, respectively.

These results can be gathered on an universal curve (Fig. 7, [18]) where the measured light production is plotted as a function of the amount of incident energy converted into ionisation of the material, $\eta(E)$, computed with the TRIM code [18].

\section{Limits from $\mathrm{CaF}_{2}$ on axially coupled WIMPs}

In this paper, we present more stringent limits on axially coupled WIMPs than the ones already available with ${ }^{73} \mathrm{Ge}$ and $\mathrm{NaI}$, due to the very favourable spin factor of Fluorine. The values we have taken in this work are from ref. [8] $\left({ }^{19} \mathrm{~F}: 0.647\right.$, $\left.{ }^{23} \mathrm{Na}: 0.041,{ }^{127} \mathrm{I}: 0.007,{ }^{73} \mathrm{Ge}: 0.065\right)$.

In order to extract limit cross-section versus WIMP mass contours, we have adopted a conservative procedure similar to the one we used in ref [5] for NaI. For a given WIMP mass, the recoil energy differential spectrum can be calculated and the lowest cross-section to which the experiment is sensitive is given by the maximum signal which can be allowed in the experimental spectrum (defined as the measured spectrum increased by 2 sigma) in any energy bin. No assumption is made on the shape of the background spectrum. 
To compare the results obtained with different nuclei, we have chosen to present all limit cross-sections as renormalized cross-sections that WIMPs would have on protons.

The expected energy recoil spectra of WIMPs on nuclei depend on three sets of assumptions: astrophysical parameters (local density, velocities), nuclear physics (matrix elements, spin factors, form factors) and instrumental effects (energy threshold, detector resolutions and scintillation efficiencies).

The instrumental factors have been measured and described in previous sections. Astrophysical assumptions and the influence of nuclear physics effects on the data are discussed in the next paragraphs.

\subsection{Astrophysical assumptions}

We make the following assumptions:

- WIMPS are distributed in a spherical halo with a local density in the solar neighbourhood, which we take to be $0.3 \mathrm{GeV} / \mathrm{cm}^{3}$.

- Their velocities follow a Maxwellian distribution in the Galactic frame truncated at a value $\mathrm{V}_{\text {esc }}$.

- We assume the WIMP average velocity $V_{r m s}$ equal to $\operatorname{sqrt}(3 / 2) V_{c}$, where $V_{c} \simeq$ $220 \mathrm{~km} / \mathrm{s}$ is the circular solar velocity.

- Transformation to the laboratory frame is computed with an Earth velocity equal to $\mathrm{V}_{c}+15 \mathrm{~km} / \mathrm{s}$, ie about $235 \mathrm{~km} / \mathrm{s}$.

Considering the uncertainty in the values to be used for the solar velocity in the Galactic halo, $V_{c}$, and the escape velocity, $V_{e s c}$, as a sensitivity check, we show in figure 8 the effects of two extreme choices on $\mathrm{Na}$ and $\mathrm{F}$ exclusion plots. The top curve for each nucleus has been calculated with $V_{c}=180 \mathrm{~km} / \mathrm{s}$ and $V_{e s c}=500 \mathrm{~km} / \mathrm{s}$; the lower curve for $V_{c}=250 \mathrm{~km} / \mathrm{s}$ and $V_{e s c}=1000 \mathrm{~km} / \mathrm{s}$.

$\mathrm{V}_{c}$ affects mainly the overall rate while the choice of $\mathrm{V}_{\text {esc }}$ affects mostly the lower mass region.

Due to the lower scintillation efficiency for Fluorine relative to Sodium, we expect Fluorine in $\mathrm{CaF}_{2}$ exclusion plots to be more sensitive than $\mathrm{Na}$ in $\mathrm{NaI}$ to the assumptions on velocities. This is shown in Fig. 8 where the limit elastic cross-sections that axially coupled WIMPS would have on protons are plotted as a function of WIMP mass.

Although the counting rate measured with the Q\&S Calcium Fluoride crystal is larger than the one obtained with our best Sodium Iodide[5], the more favourable spin factor of Fluorine allows us to set an overall better limit for this target nucleus than for $\mathrm{Na}$, for WIMP masses above $20 \mathrm{GeV}$. 


\subsection{Comparison with results on other target nuclei}

In the calculation of the rates, we have included the spin factors calculated in the odd group model of Ellis and Flores [8], and a spin form factor with a Bessel function shape as for the mass form factor of Engel (1991) in reference [9]. Ellis and Flores (1991) suggested a correction for the spin radius with respect to the charge radius which they tabulated in ref [8] for different nuclei. This is taken into account. The spin form factor introduces a negligible correction for $\mathrm{Na}$ and $\mathrm{F}$.

In Fig. 9 we show exclusion plots for various detector nuclei for which data are available. Here, the WIMP circular velocity in the Galactic frame is taken to be the solar circular velocity $V_{c}=220 \mathrm{~km} / \mathrm{s}$, the Earth velocity is $235 \mathrm{~km} / \mathrm{s}$, and the escape velocity in the laboratory frame is chosen to be $800 \mathrm{~km} / \mathrm{s}$. The scintillation/ionisation efficiencies used are respectively $0.25,0.07,0.069$, and 0.3 for $\mathrm{Na}, \mathrm{I}$, $F$, and ${ }^{73} \mathrm{Ge}$.

The exclusion plots drawn in Fig. 9a are evaluated with the form factors described above.

For ${ }^{73} \mathrm{Ge}$, the data are from ref. [4]; we should point out that an additional source of difficulty in comparing these data with other nuclei, arises from the fact that its unpaired nucleon is a neutron, while for the other 3 nuclei, the unpaired nucleon is a proton.

For comparison, the limits obtained without any form factors are also shown on figure $9 \mathrm{~b}$. The differences are clear in the case of heavy nuclei.

The important point is however, that Fluorine gives always better limits than other nuclei for axial couplings and is not sensitive to assumptions on the spin form factor.

\section{Conclusion}

In conclusion, we presented here results on CDM search with a new scintillator improving the exclusion plots on axially coupled particles. We plan to improve the anti-Compton rejection and to consider purification possibilities of the detector material to reach counting rates that will allow us to approach the neutralino region.

\section{Acknowledgements}


It is a pleasure to thank Dr. C. Arpesella for valuable support, Mr A. Bussolotti for technical help and the LNGS and LSM staff for support.

\section{Figure captions}

Figure 1: The ${ }^{55} \mathrm{Fe}$ source as seen by the $0.370 \mathrm{Kg} \mathrm{Q} \& \mathrm{~S}$ detector; the hardware threshold is $1.9 \mathrm{keV}$.

Figure 2: Experimental rate for our $0.370 \mathrm{Kg}$ detector from Quartz and Silice.

Figure 3: High energy spectrum for the Merck crystal (same mass).

Figure 4: Decay time of the pulse as a function of the energy for the events obtained with the Merck crystal.

Figure 5 : Spectra obtained for recoil ions of a) ${ }^{19} \mathrm{~F}$ at $\mathrm{E}_{n}=1.3 \mathrm{MeV}$ and b) $\mathrm{Ca}$ and ${ }^{19} \mathrm{~F}$ at $\mathrm{E}_{n}=1.9 \mathrm{MeV}$ for $\theta=115^{\circ}$.

Figure 6 : Energy produced in the crystal - in keV electron equivalent units (kee)as a function of kinetic energies of ${ }^{19} \mathrm{~F}$ and $\mathrm{Ca}$ ions.

Figure 7 : Measured light production (in kee) as a function of $\eta(\mathrm{E})$.

Figure 8: Exclusion plots for axially coupled WIMPs derived from our measurements in the case of two extreme hypothesis for $V_{\text {halo }}$ and $V_{\text {esc }}$ (cf text).

Figure 9: Exclusion plots for axially coupled WIMPs: a) considering the form factor described in the text; b) without any form factor. Exclusion plots on $\mathrm{Na}, \mathrm{I}$ [5], and ${ }^{73} \mathrm{Ge}[4]$ are shown for comparison.

\section{References}

1. P. F. Smith and J.D. Lewin, Phys. Rep. 187 (1990), 203.

2. S. P. Ahlen et al., Phys. Lett. B195 (1987), 603.

3. D. O. Caldwell et al., Phys. Rev. Lett. 61 (1988), 510.

4. D. Reusser et al., Phys. Lett. B255 (1991),143.

5. C. Bacci et al., Phys. Lett. B293 (1992), 460.

6. A. Bottino et al., Phys. Lett. B295 (1992), 330.

7. P. Belli et al., Nucl. Instr. \& Meth. A336 (1993), 336.

8. J. Ellis and R.A. Flores, Phys. Lett. B263 (1991), 259.

9. J. Engel, PL B264 (1991), 114.

Engel, Pittel, Vogel, Int. J. Mod. Phys. E1 (1992) 1.

10. E. Bellotti, Nucl. Instr. \& Meth. A264 (1988), 1.

11. G. Gerbier, to appear in the proceedings of the workshop "The dark side of the Universe: experimental efforts and theoretical framework", Roma "Tor Vergata", June 1993.

12. Technical note of Harshaw/Q\&S

13. G. Gerbier et al., Phys. Rev D 42 (1990) 3211 ;

P. Zecher et al. Phys.Rev A41 (1990) 4058.

14. C. Chasman et al. Phys. Rev. Lett.21 (1968) 1431. 
15. G. Gerbier et al. to be published.

16. "Neutron cross-sections", V. Mc.Lane, C.L. Dunford, P.F. Rose Academic Press, inc. 1250, sixth Avenue, San Diego CA 92101.

17. G.L. Cano and G.S. Lockwood Phys. Rev. 157 (1967) 677.

18. "The stopping and range of ions in solids" J.F. Ziegler, J.P. Biersack, U. Littmark. 1985 Pergamon Press, Inc. 


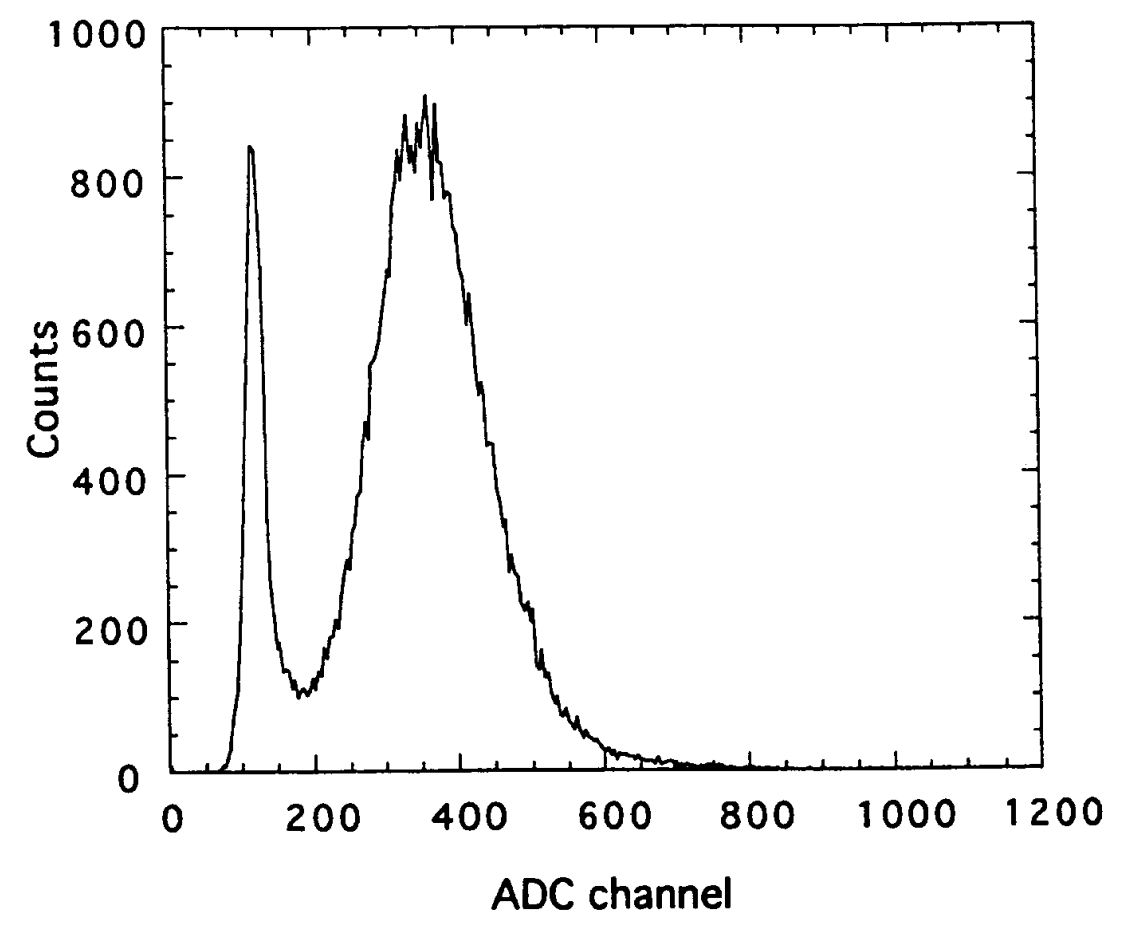

Figure 1 


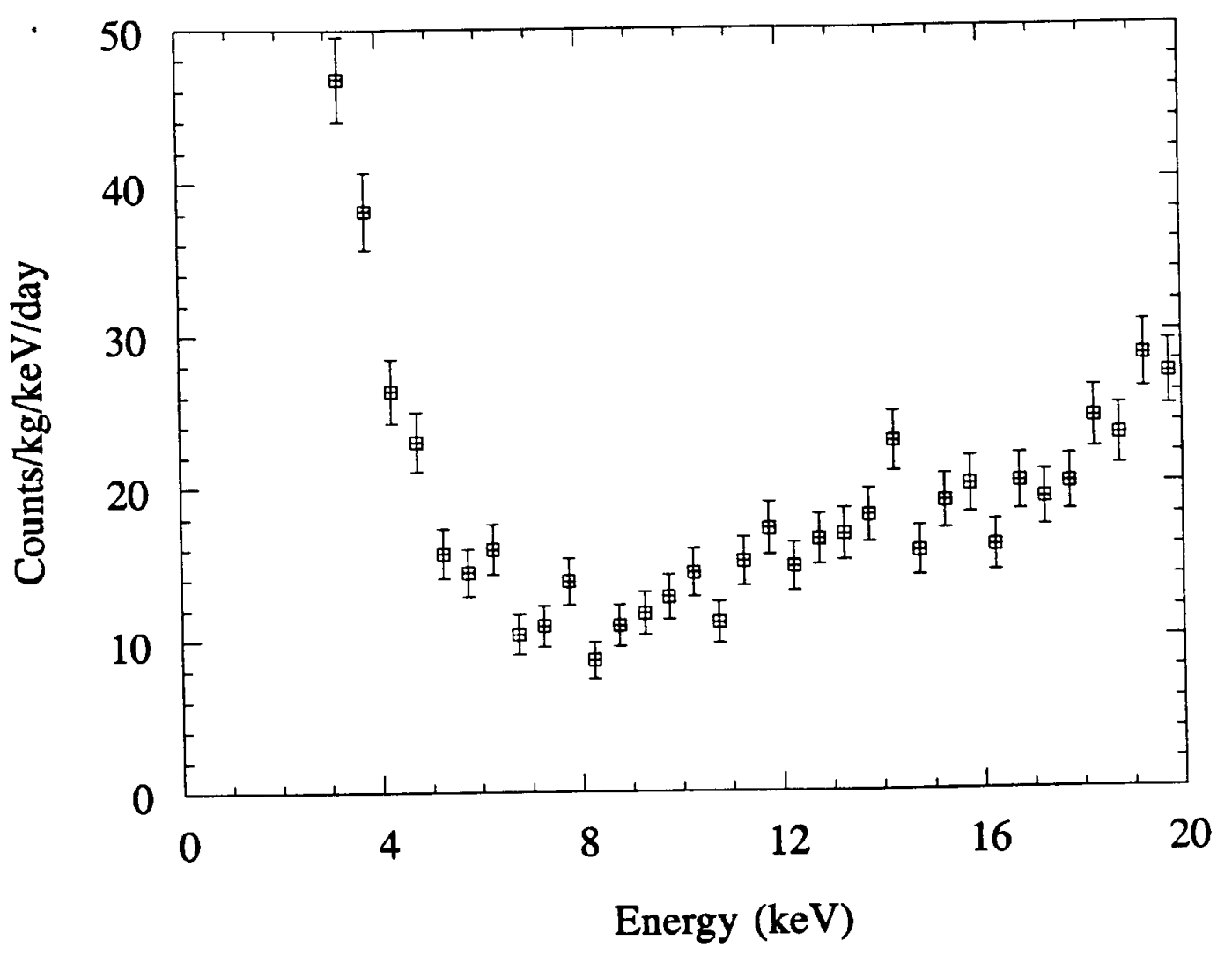

Figure 2 


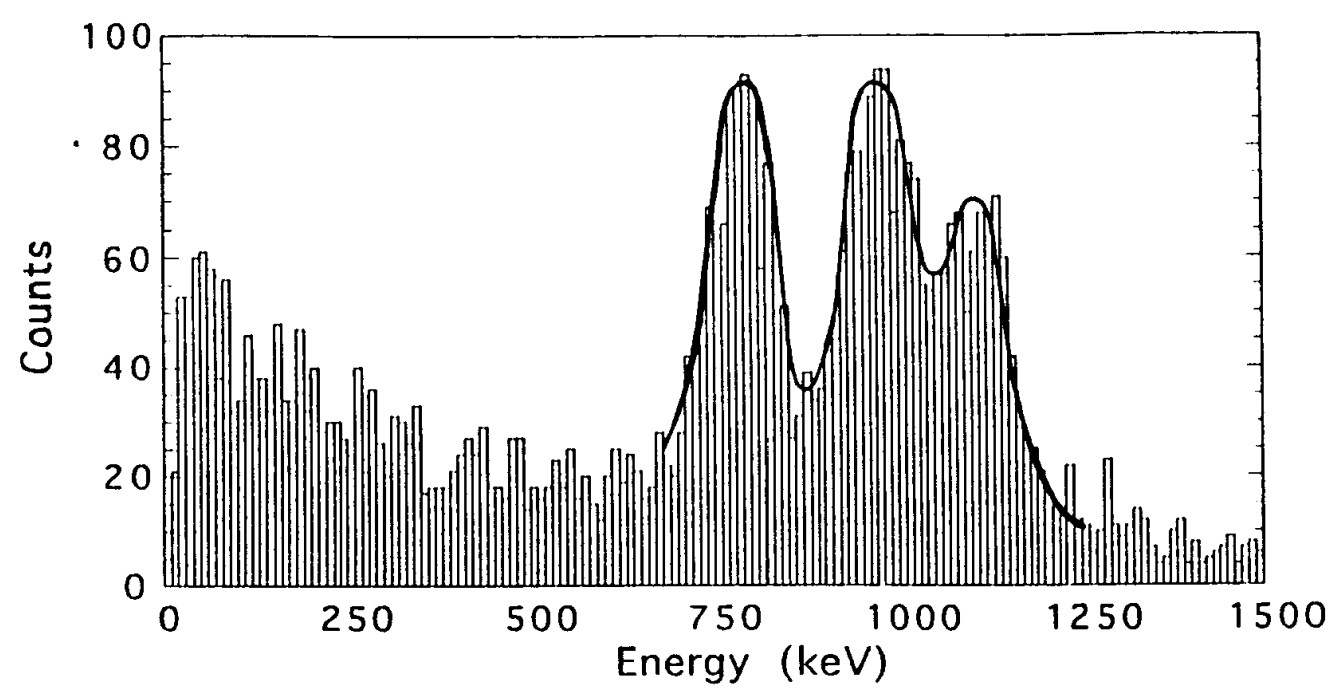

Figure 3

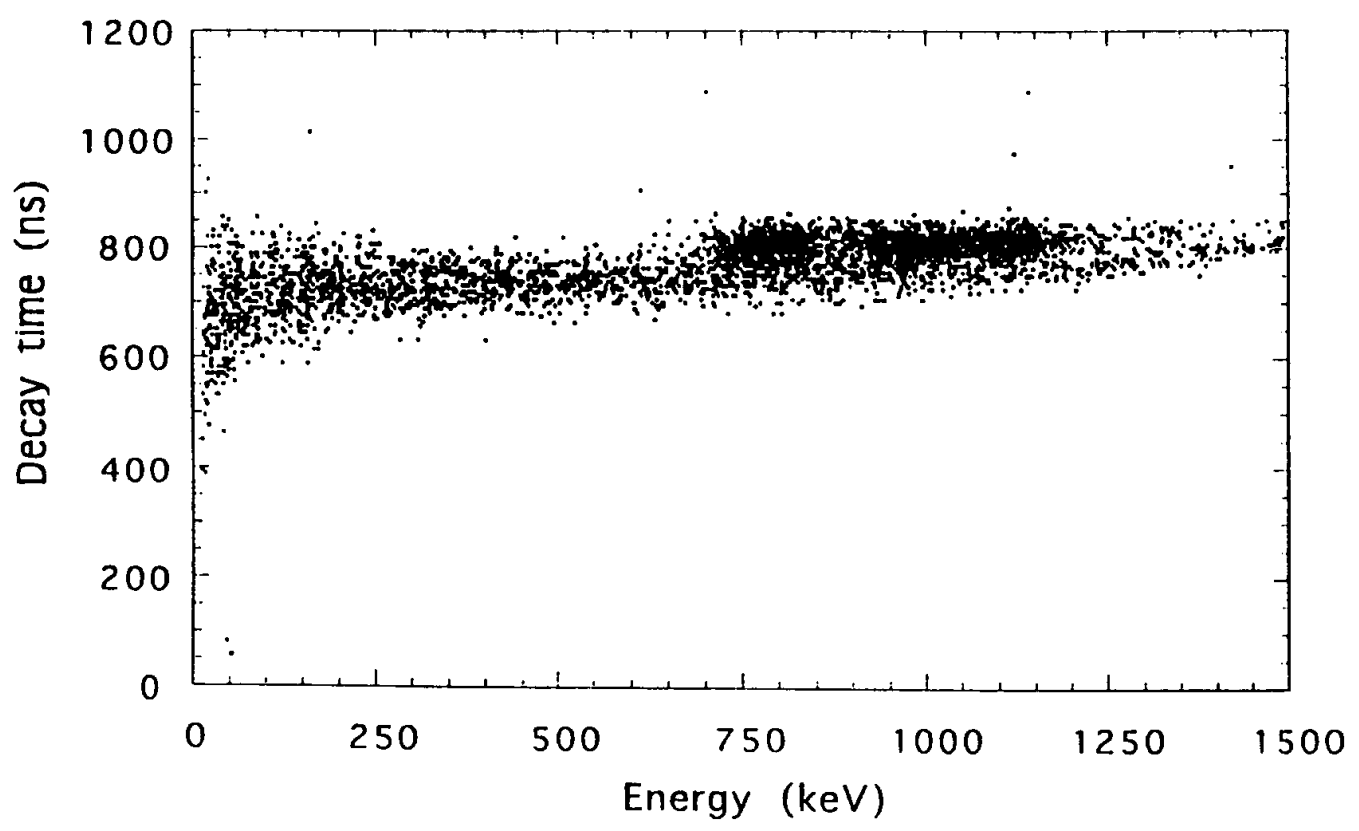

Figure 4 


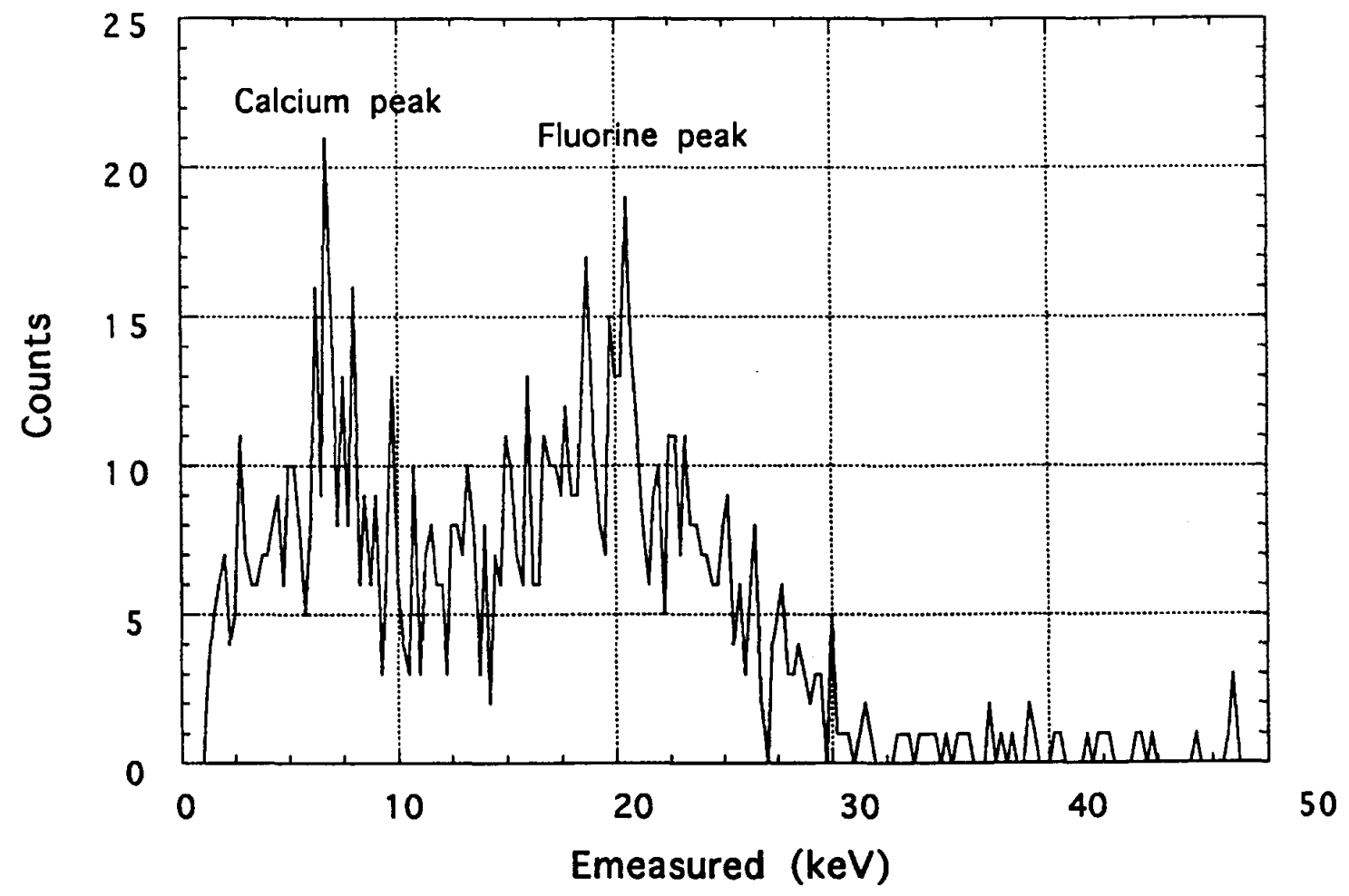

Figure 5 


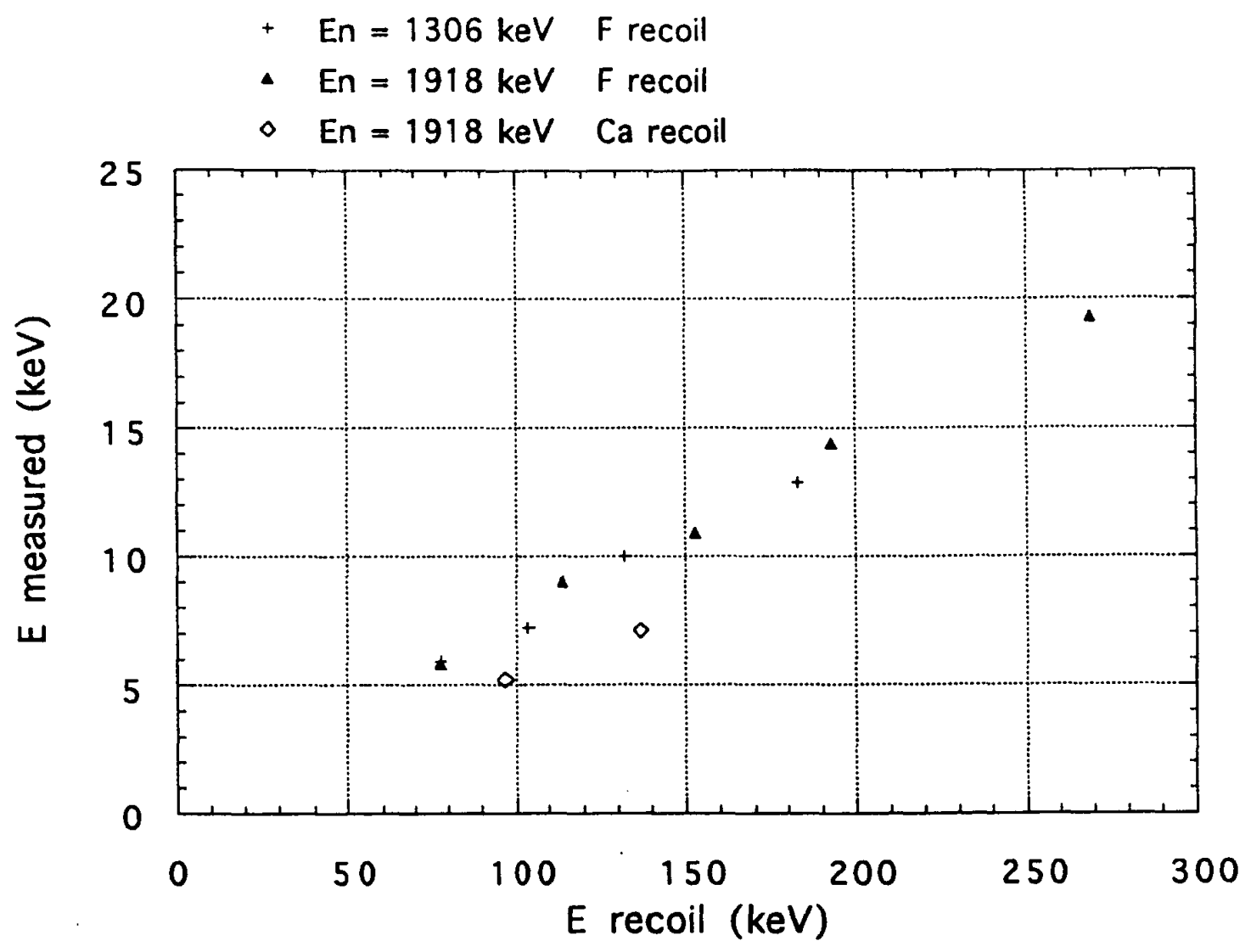

Figure 6

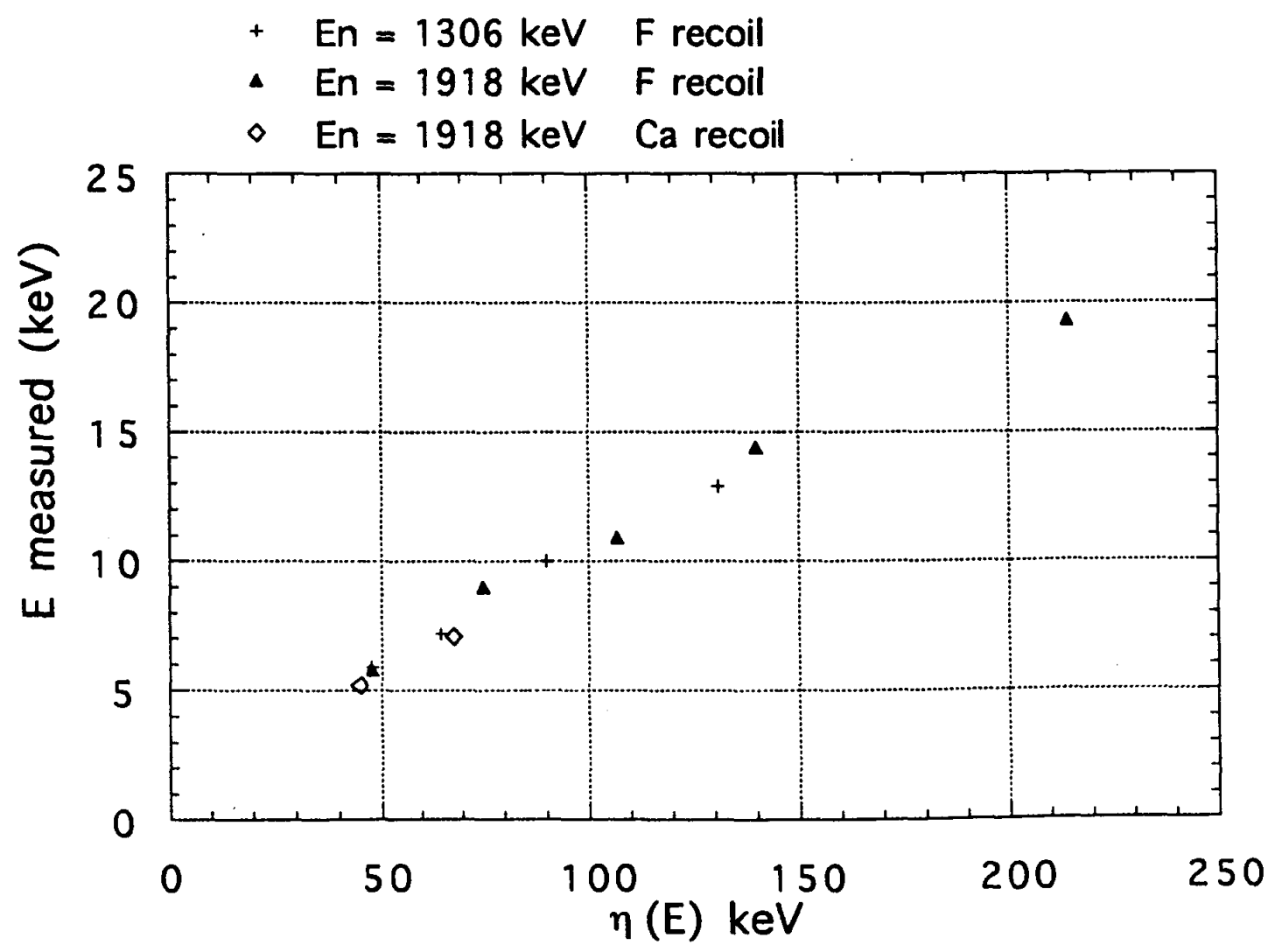

Figure 7 


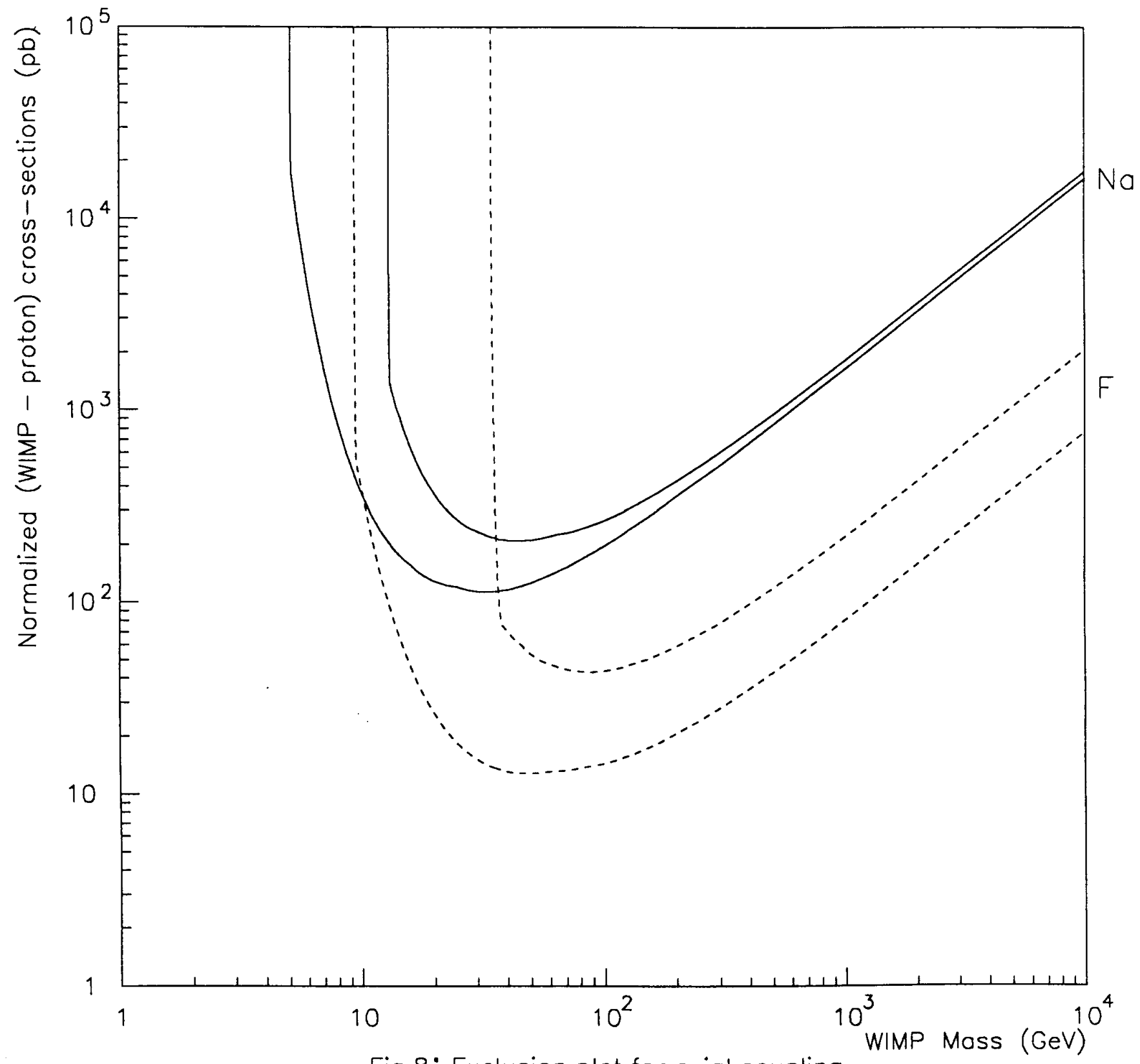

Fig 8: Exclusion plot for axial coupling 


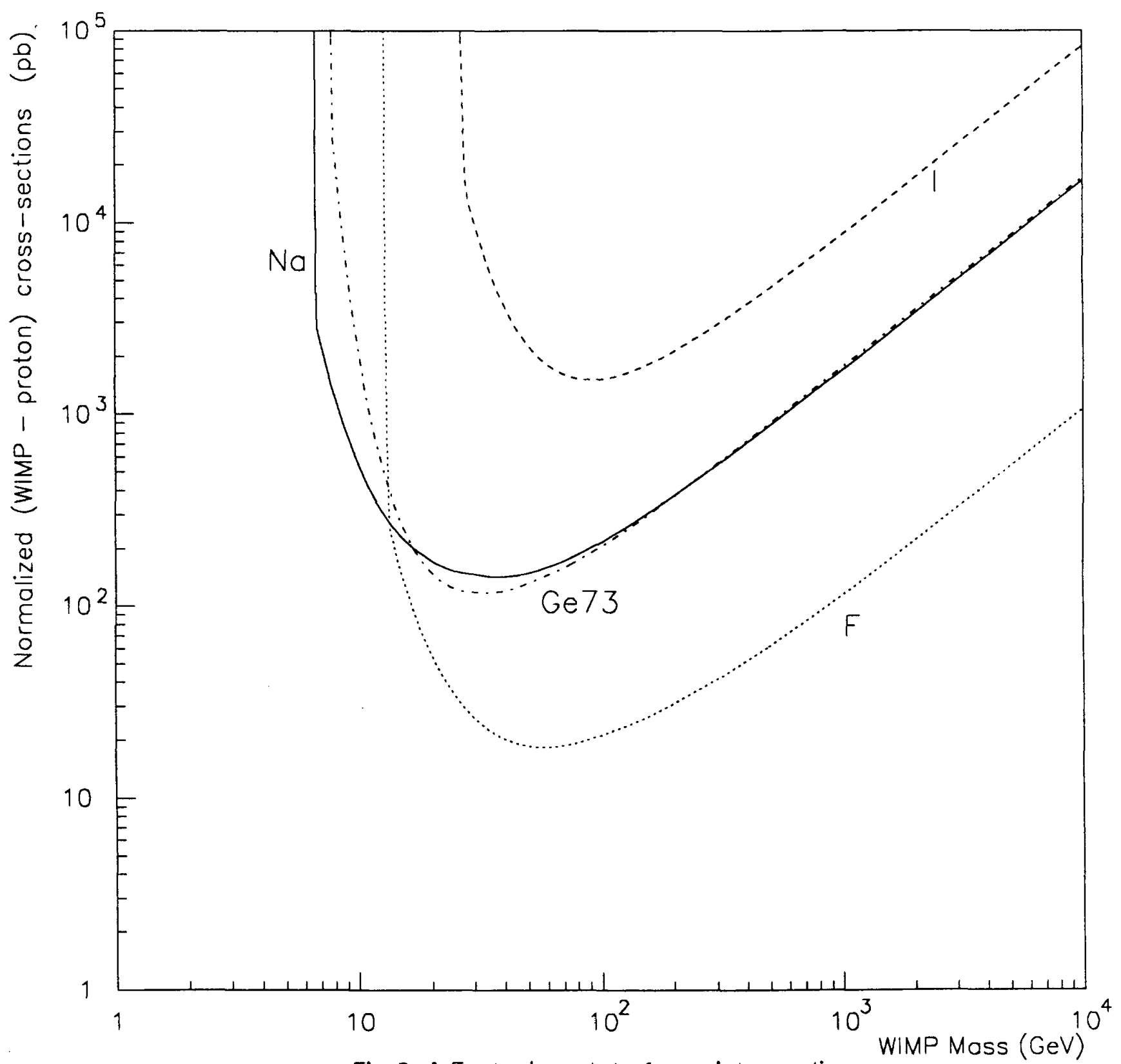

Fig 9a: Exclusion plots for axial coupling 


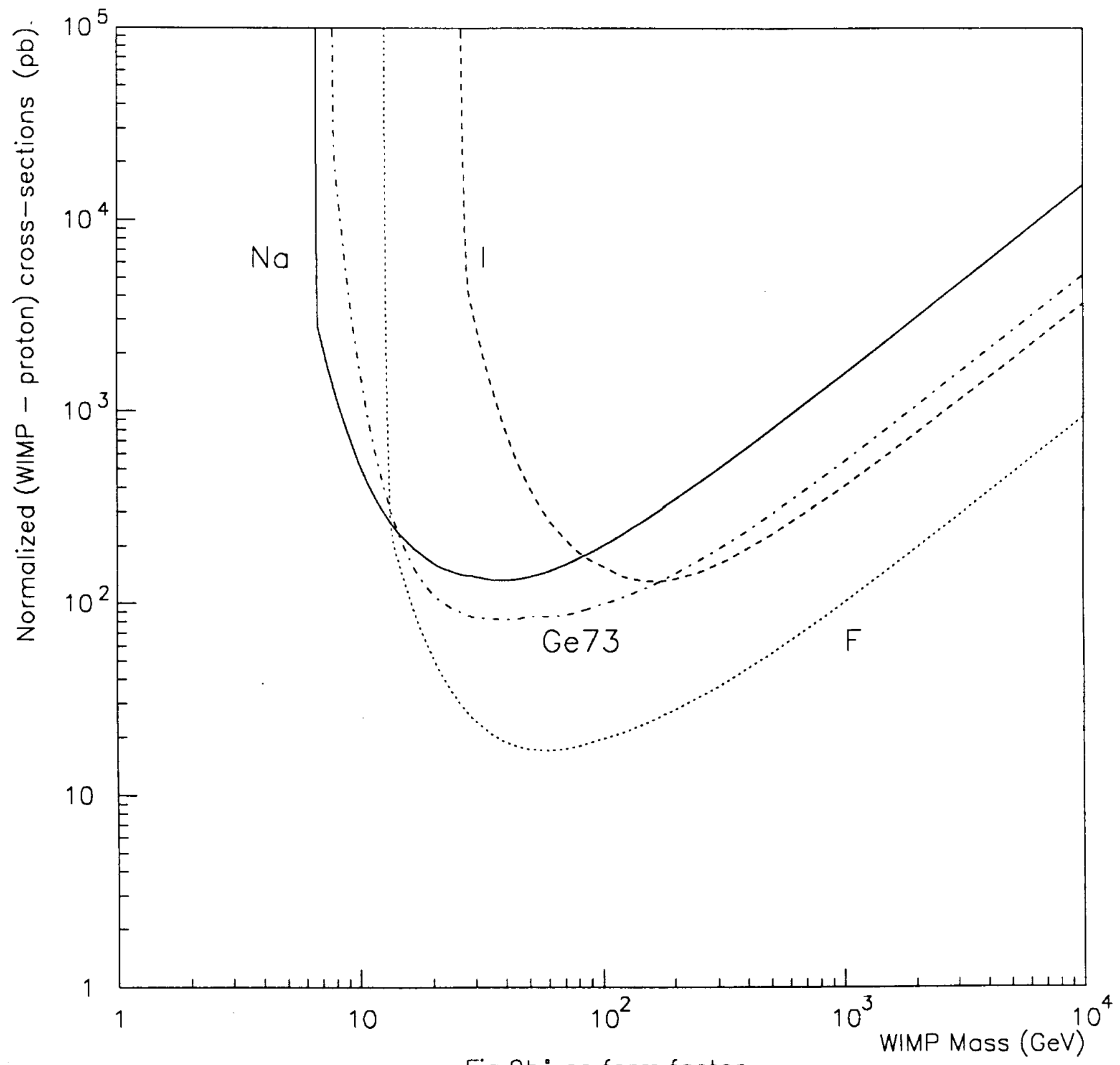

Fig 9b: no form factor 
